\title{
基于 Hanson 噪声模型的螺旋桨气动与 噪声优化设计
}

\author{
宋翔, 余培汛, 白俊强, 韩啸, 彭嘉辉
}

(西北工业大学 航空学院, 陕西 西安 710072)

\begin{abstract}
摘 要: 针对螺旋桨气动与噪声多目标优化设计问题,采用基于非均匀有理 $\mathrm{B}$ 样条的自由曲面变形方 法对全桨叶进行三维几何变形。为节省优化计算成本,将 RANS 方法和 Hanson 模型相结合预测纯音 噪声, 其预测精度与耦合 URANS 方法的 FW-H 方程相当。在此基础上,采用 Kriging 代理模型与非支 配关系排序遗传算法进行优化搜索,建立了螺旋桨气动与噪声多目标优化设计框架。采用该框架对 某民航客机螺旋桨进行优化设计,优化以叶片不同展向站位的翼型扭转角和弦长作为设计变量。相 比基础桨叶, 在功率不增加的情形下, 巡航构型风洞试验状态的轴向监测点噪声值最大下降约 $0.25 \mathrm{~dB}$, 在功率略有增加的情形下, 喿声降低约 $1 \mathrm{~dB}$ 。
\end{abstract}

关 键 词: 自由曲面变形方法 (FFD); 多重参考坐标系 (MRF); Hanson 噪声模型; 非支配关系排 序算法 (NSGAII)

中图分类号: V211.4 文献标志码: A

螺旋桨推进装置因为其低空、低马赫飞行下高 的推进效率、出色的起降性能及经济性好等优势, 广 泛应用于中小型运输机、舰载预警机、支线客机等航 空飞行器上。随着化石能源的日益枯竭和人们对环 境污染问题的日益关注, 对比喷气式推进装置, 高推 进效率、低燃油消耗的螺旋桨推进装置得到越来越 多的重视,迎来了发展的新机遇。然而, 噪声污染问 题一直是螺旋桨飞机发展的一大挑战。螺旋桨噪声 除了影响驾驶员和乘客的舒适度、对机场附近形成 严重的噪声污染外, 在极端情况下, 会诱发结构的振 动和声疲劳问题, 影响飞机的安全性。因此, 控制螺 旋桨辐射噪声是现代飞行器设计必须要面对的重要 问题, 开展螺旋桨噪声预测及控制研究工作具有重 要的现实意义。

在螺旋桨噪声预测及控制的研究工作中, 针对 低噪声螺旋桨设计问题, 国外已经开展了大量的研 究工作。Pagano 等 ${ }^{[1]}$ 对 P180 飞机螺旋桨桨叶进行 了低噪声设计, 在六叶桨的基础上, 进行了考虑气动
文章编号: 1000-2758(2020)04-0685-10

性能、气动弹性和气动噪声的多目标优化设计, 获得 了 $1.5 \mathrm{~dB}$ 的降噪量。Marinus 等 ${ }^{[2]}$ 利用 Fluent 软 件、FW-H 方程和 Samcef 软件对气动、噪声和结构强 度进行评估, 采用改进的粒子群算法进行了优化设 计。优化构型在起飞着陆阶段获得了 $5.2 \mathrm{~dB}$ 的降 噪量, 在巡航阶段, 获得了 $7.7 \mathrm{~dB}$ 的降噪量。在不 改变发动机型号、气动性能损失小于 $3 \%$ 的前提下, Canard 等 ${ }^{[3]}$ 优化得到的 ANIBAL 桨叶巡航阶段的性 能略差于初始桨, 但噪声降低了 $7 \mathrm{~dB}$ 。国内王 博 ${ }^{[4]}$ 、招启军等 ${ }^{[5]}$ 研究工作者陆续开展了基于 CFD 的螺旋桨或是旋翼的优化设计, 但关于螺旋桨气动 与噪声优化设计的研究工作相对较少, 郭旺柳等 ${ }^{[6]}$ 采用 URANS 方程和 FW-H 方程分别计算流场和声 场, 结合 Kriging 代理模型和遗传算法针对旋翼桨尖 外形进行了低噪声设计, 获得了 $5 \mathrm{~dB}$ 的降噪量。朱 正等 ${ }^{[7]}$ 结合 RANS、FW-H 方程、代理模型和遗传算 法对旋翼桨尖外形进行了多目标优化, 优化构型显 著降低了桨尖浴强度和旋翼声压峰值。 
综合看来, 国内螺旋桨气动与噪声多目标优化 设计研究主要针对旋翼桨尖外形, 且大多采用二次 函数或者三次函数对桨尖前后缘曲线等进行参数 化, 难以直接对螺旋桨的三维曲面进行几何变形和 重构。本文采用自由曲面变形和基于径向基函数插 值的动网格方法对全桨进行优化, 外形参数化方法 更为直观、灵活和鲁棒。此外, 采用 Ffows WilliamsHawings ( FW-H ) 方程进行噪声求解, 为了保证足够 高的计算精度, 一般需要求解非定常流场, 计算成本 高。为了准确预测螺旋桨噪声的同时节约计算成 本, 许多针对螺旋桨噪声预测的频域计算方法发展 起来。Gutin ${ }^{[8]}$ 在 1932 年提出了第一个成功应用于 螺旋桨噪声计算的声学模型, 但该模型仅适用于只 有轴向流动存在情形下无后掠简单外形、低叶尖马 赫数、无前进速度的螺旋桨。Deming ${ }^{[9]}$ 第一个通过 叠加螺旋桨平面中连续的源和汇的环模拟了有限厚 度的叶片引起的噪声, 但并没有有效地消除声学理 论上的限制。1953 年, Garrick 和 Watkins ${ }^{[10]}$ 扩展了 推力和扭矩引起的载荷噪声研究, 考虑了亚声速前 进飞行的影响。1956 年, Arnoldi ${ }^{[11-12]}$ 进一步发展了 前向飞行时螺旋桨厚度噪声理论。1971 年, Barry 和 Magliozzi $^{[13]}$ 完善了 Garrick 及 Watkins 和 Arnoldi 的载荷噪声和厚度噪声的理论, 并考虑了叶片扭转 的影响。1980 年, Hanson ${ }^{[14]}$ 在此基础上提出了不 局限于低叶尖马赫数要求、可适用于带后掠螺旋桨 前向飞行的噪声预测模型。随后, Hanson ${ }^{[15]}$ 继续发 展该模型并将其应用到迎角不为零飞行时螺旋桨噪 声预测问题。Kotwicz 等于 2017 年 ${ }^{[16]}$ 和 2019 年 ${ }^{[17]}$, 通过与多个模型实验结果对比, 研究了不同 螺旋桨噪声模型的预测精度。结果表明在预测纯音 噪声时, 叶素动量理论结合 Hanson 噪声模型的计算 方式相对于其他早期噪声理论, 多个算例与实验的 平均误差最小 ${ }^{[8]}$, 具有较高的计算精度。因为叶素 动量理论假设叶素上的作用力只与叶素所在圆环内 的流体动量变化相关, 忽略了相邻圆环流体之间展 向的相互作用,所以本文采用计算精度相对更高的 定常 RANS 方程计算方法, 将 RANS 方程与 Hanson 相结合。相对于 URANS 结合 FW-H 方程的方法, 该 方法在保证较高精度的同时计算成本相对较低, 且 能较为快速计算监测点的载荷噪声和厚度噪声。

在此基础上,本文结合 Kriging 代理模型和遗传 算法, 建立了螺旋桨多目标优化平台, 并对某民航客 机螺旋桨进行了降噪优化, 获得了一些有意义的
结论。

\section{1 桨叶参数化与网格变形}

\section{1 自由曲面变形方法}

螺旋桨桨叶外形参数化是优化设计的基础, 本 文采用基于非均匀有理 B 样条 (non-uniform Bspline, NURBS) 的自由曲面变形 (free form deformation,FFD) 方法 (NFFD), 对初始构型进行展向各个 剖面的扰动, 继而得到优化过程中的构型。自由曲 面变形方法最初是由 Sederberg 和 Parryin ${ }^{[18]}$ 在 1986 年提出的,其变形过程可以看作由 2 个部分组成。 首先, 控制点以给定的方式移动。其次, 研究对象根 据函数关系随控制点的移动而变形。在数学中, 在 通过移动控制点变形之前, 需要将嵌人在 FFD 框架 中的点映射到参数空间, 创建研究对象和控制点坐 标之间的函数关系, 如(1) 式所示

$$
\boldsymbol{X}(s, t, u)=\sum_{i=0}^{l} \sum_{j=0}^{m} \sum_{k=0}^{n} \boldsymbol{P}_{i, j, k} B_{i l}(s) B_{j m}(t) B_{k n}(u)
$$

式中: $\boldsymbol{X}(s, t, u)$ 和 $\boldsymbol{P}_{i, j, k}$ 分别为研究对象表面上任意 点的坐标和控制点的坐标; $s, t$ 和 $u$ 为参数空间中表 面几何点的局部坐标, 该数值由初始构型表面几何 点和 FFD 控制点坐标确定, 即求解 (1) 式方程组得 到; $(l+1),(m+1)$ 和 $(n+1)$ 为控制框 3 个方向的 控制点数; $B_{i l}(s), B_{j m}(u)$ 和 $B_{k n}(t)$ 为 Bernstein 多项 式, 表示为

$$
B_{i l}(s)=\frac{l !}{i !(l-i) !} s^{i}(l-s)^{(l-i)}
$$

在通过移动控制点使得控制框变形后, 可以得 到控制点的坐标位移 $\Delta \boldsymbol{P}_{i, j, k}$ 。将 $\Delta \boldsymbol{P}_{i, j, k}$ 代人 (2) 式 即可得到研究对象表面几何点的坐标位移 $\boldsymbol{s}_{b}$ 。

$$
\begin{gathered}
\boldsymbol{s}_{b}=\Delta \boldsymbol{X}(s, t, u)= \\
\sum_{i=0}^{l} \sum_{j=0}^{m} \sum_{k=0}^{n} \Delta \boldsymbol{P}_{i, j, k} B_{i l}(s) B_{j m}(t) B_{k n}(u)
\end{gathered}
$$

显然,变形后的几何上任意点的坐标可以表示为

$$
\boldsymbol{X}^{\prime}(s, t, u)=\boldsymbol{X}(s, t, u)+\boldsymbol{s}_{b}
$$

然而,采用基于 Bernstein 多项式的原始 FFD 方 法, 控制点的位移将导致控制框内几何的整体变化。 为了增强对研究对象变形的控制, NFFD 方法 ${ }^{[19]}$ 采 用了非均匀有理 B 样条构建控制框, 并引人了控制 点的权因子, 不仅可以通过调整控制点位置而且可 以通过改变控制点的权因子来改变几何外形。数学 
上, NFFD 方法利用 NURBS 基函数替换了 Bernstein 公式,如(4)式所示

$$
\begin{gathered}
\boldsymbol{X}(s, t, u)= \\
\frac{\sum_{i=0}^{l} \sum_{j=0}^{m} \sum_{k=0}^{n} \boldsymbol{P}_{i, j, k} W_{i, j, k} B_{i, p}(s) B_{j, q}(t) B_{k, r}(u)}{\sum_{i=0}^{l} \sum_{j=0}^{m} \sum_{k=0}^{n} W_{i, j, k} B_{i, p}(s) B_{j, q}(t) B_{k, r}(u)}
\end{gathered}
$$

式中: $W_{i, j, k}$ 为控制点的权因子; $B_{i, p}(s), B_{j, q}(u)$ 和 $B_{k, r}(t)$ 分别为 $p$ 阶, $q$ 阶和 $r$ 阶的有理 $\mathrm{B}$ 样条基 函数。

\section{2 基于径向基函数的网格变形方法}

为了评估其气动性能, 需要生成新构型的网格 用于数值模拟。本文选择使用径向基函数 (radial basis function, RBF) 进行网格变形。利用 RBF 实现 网格变形的方法首先由 Boer 等 ${ }^{[20]}$ 提出, 基于 $\mathrm{RBF}$ 的网格变形方法仅需要初始网格坐标和表面网格点 位移, 可以容易地实现新网格自动生成。用于表示 网格点位移的插值函数可写为

$$
\boldsymbol{s}(\boldsymbol{x})=\sum_{i=1}^{N} \gamma_{i} \varphi\left(\left\|\boldsymbol{x}-\boldsymbol{x}_{i}\right\|\right)
$$

式中: $\boldsymbol{s}(\boldsymbol{x})$ 为任意网格点坐标 $\boldsymbol{x}$ 处的位移, $N$ 为表 面网格点的数目; $i$ 为表面网格点的编号; $\gamma_{i}$ 和 $\boldsymbol{x}_{i}$ 分 别为第 $i$ 个网格点处的插值系数和坐标; $\varphi(\| \boldsymbol{x}-$ $\boldsymbol{x}_{i} \|$ ) 为径向基函数。为了确定权重系数 $\gamma_{i}$, 将表 面网格点坐标和位移代人 (6) 式, 可以得到关于权 重系数的方程组

$$
\boldsymbol{s}_{b}\left(\boldsymbol{x}_{b}\right)=\left[\begin{array}{c}
\Delta \boldsymbol{x}_{b} \\
\Delta \boldsymbol{y}_{b} \\
\Delta \boldsymbol{z}_{b}
\end{array}\right]=\left[\begin{array}{c}
\boldsymbol{\Phi}_{b} \boldsymbol{\gamma}_{x, b} \\
\boldsymbol{\Phi}_{b} \boldsymbol{\gamma}_{y, b} \\
\boldsymbol{\Phi}_{b} \boldsymbol{\gamma}_{z, b}
\end{array}\right]
$$

式中

$$
\begin{aligned}
& \Delta \boldsymbol{x}_{b}=\left[\Delta x_{b 1}, \Delta x_{b 2}, \cdots, \Delta x_{b N}\right]^{\mathrm{T}} \\
& \Delta \boldsymbol{y}_{b}=\left[\Delta y_{b 1}, \Delta y_{b 2}, \cdots, \Delta y_{b N}\right]^{\mathrm{T}} \\
& \Delta \boldsymbol{z}_{b}=\left[\Delta z_{b 1}, \Delta z_{b 2}, \cdots, \Delta z_{b N}\right]^{\mathrm{T}} \\
& \boldsymbol{\gamma}_{x, b}=\left[\gamma_{x, b 1}, \gamma_{x, b 2}, \cdots, \gamma_{x, b N}\right]^{\mathrm{T}} \\
& \boldsymbol{\gamma}_{y, b}=\left[\gamma_{y, b 1}, \gamma_{y, b 2}, \cdots, \gamma_{y, b N}\right]^{\mathrm{T}} \\
& \boldsymbol{\gamma}_{z, b}=\left[\gamma_{z, b 1}, \gamma_{z, b 2}, \cdots, \gamma_{z, b N}\right]^{\mathrm{T}}
\end{aligned}
$$

此处, $s_{b}$ 为表面网格点位移组成的矩阵, 物面边界 网格位移可由 (2) 式得到, 远场边界表面网格位移 设置为零。 $\Delta x_{b}, \Delta y_{b}$ 和 $\Delta z_{b}$ 为 3 个方向的位移矩阵, $\boldsymbol{\gamma}_{x, b}, \boldsymbol{\gamma}_{y, b}$ 和 $\gamma_{z, b}$ 为插值系数, $\Phi_{b}$ 为表面网格点径向 基函数值 $\varphi_{b i b j}=\varphi\left(\left\|\boldsymbol{x}_{b i}-\boldsymbol{x}_{b j}\right\|\right)$ 组成的矩阵, 具体 形式如下

$$
\boldsymbol{\Phi}_{b}=\left[\begin{array}{cccc}
\varphi_{b 1 b 1} & \varphi_{b 1 b 2} & \cdots & \varphi_{b 1 b N} \\
\vdots & \vdots & \ddots & \vdots \\
\varphi_{b N b 1} & \varphi_{b N b 2} & \cdots & \varphi_{b N b N}
\end{array}\right]_{N \times N}
$$

求解方程组(6) 得到权重系数后, 将空间网格 点坐标代人 (7) 式中, 即可得到空间网格点位移

$$
\boldsymbol{s}_{v}\left(\boldsymbol{x}_{v}\right)=\left[\begin{array}{c}
\Delta \boldsymbol{x}_{v} \\
\Delta \boldsymbol{y}_{v} \\
\Delta \boldsymbol{z}_{v}
\end{array}\right]=\left[\begin{array}{l}
\boldsymbol{\Phi}_{v} \boldsymbol{\gamma}_{x, b} \\
\boldsymbol{\Phi}_{v} \boldsymbol{\gamma}_{y, b} \\
\boldsymbol{\Phi}_{v} \boldsymbol{\gamma}_{z, b}
\end{array}\right]=\left[\begin{array}{l}
\boldsymbol{\Phi}_{v} \boldsymbol{\Phi}_{b}^{-1} \Delta \boldsymbol{x}_{b} \\
\boldsymbol{\Phi}_{v} \boldsymbol{\Phi}_{b}^{-1} \Delta \boldsymbol{y}_{b} \\
\boldsymbol{\Phi}_{v} \boldsymbol{\Phi}_{b}^{-1} \Delta \boldsymbol{z}_{b}
\end{array}\right]
$$

则新网格点的坐标即可通过网格点位移加上初始网 格坐标得到。

\section{3 初始桨叶外形及参数化变量}

优化选用的初始构型为某客机的风洞实验模 型, 螺旋桨直径 $D$ 为 $0.737 \mathrm{~m}$, 为真实模型的 $1 / 6$, 如 图 1 所示。为了实现变形, 布置了由 8 个与叶尖端 面平行的截面组成的控制框。从叶根到叶尖, 8 个 截面分别位于 $r / R=0.225,0.315,0.45,0.586,0.721$, $0.811,0.901$ 和 1.0 的展向位置处。设计的控制框 如图 2 所示。

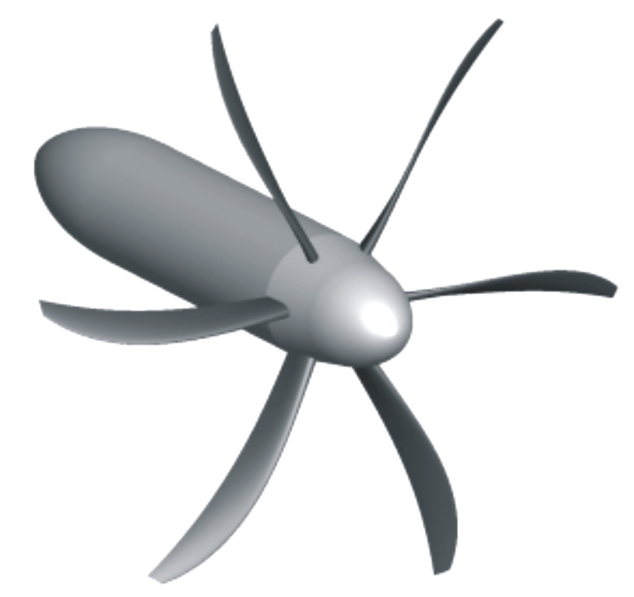

图 1 初始螺旋桨

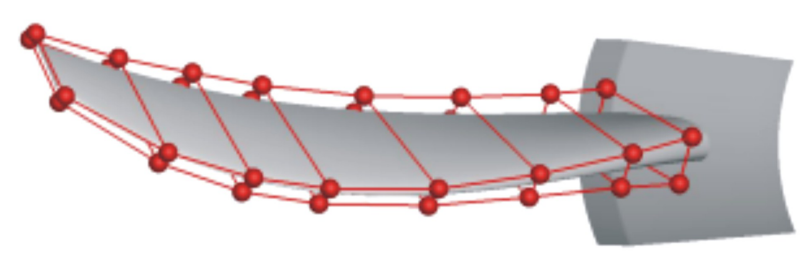

图 2 包含初始叶片的控制框

在叶片变形过程中, 中间 6 个截面分别以当地 翼型弦线中点为中心沿弦线方向放缩, 和以中心在 螺旋桨旋转轴上投影点与中心的连线为旋转轴旋 转。优化共有 12 个设计变量, 即为各个截面的放缩 因子和扭转角。为了验证方法的可行性, 随机生成 
设计变量数值, 对控制框和叶片进行扰动。图 3 为 变形前后控制框的对比图, 实线和球体表示初始控 制框的边线和节点, 虚线和八面体表示变形后控制 框几何。图 4 为变形前后叶片的几何对比图, 图 4a) 为初始叶片, 图 4b) 为变形后叶片。

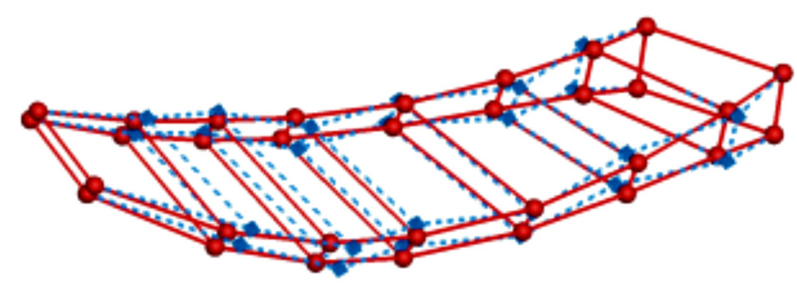

图 3 变形后控制框和初始控制框对比

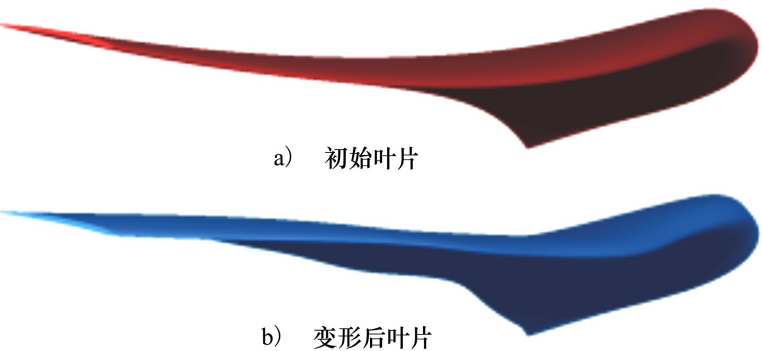

图 4 变形后叶片与初始叶片对比

\section{2 性能评估及优化算法}

\section{1 气动评估}

螺旋桨的气动性能通过多重参考坐标系 (multiple reference frame, MRF) 方法评估, 该方法可 以模拟涉及相对运动的流动, 具有较高的模拟精 度 ${ }^{[21]}$ 。旋转参考系中的 Navier-Stokes 如(8) 式所示

$$
\begin{aligned}
\frac{\partial}{\partial t} \iiint_{V} \boldsymbol{Q} \mathrm{d} V+\iint_{\partial V} \boldsymbol{F} \cdot \boldsymbol{n} \mathrm{d} S- \\
\iint_{\partial V} \boldsymbol{F}_{v} \cdot \boldsymbol{n} \mathrm{d} S+\iiint_{V} \boldsymbol{G} \mathrm{d} V=0
\end{aligned}
$$

式中, $\boldsymbol{Q}, \boldsymbol{F}, \boldsymbol{F}$ 和 $\boldsymbol{G}$ 分别为守恒变量、无黏通量、黏 性通量和源项。具体形式如下

$$
\boldsymbol{Q}=\left[\begin{array}{c}
\rho \\
\rho u \\
\rho v \\
\rho w \\
\rho E
\end{array}\right] \quad \boldsymbol{F}=\left[\begin{array}{c}
\rho\left(\boldsymbol{q}-\boldsymbol{q}_{b}\right) \\
\rho u\left(\boldsymbol{q}-\boldsymbol{q}_{b}\right)+p \boldsymbol{n}_{x} \\
\rho v\left(\boldsymbol{q}-\boldsymbol{q}_{b}\right)+p \boldsymbol{n}_{y} \\
\rho w\left(\boldsymbol{q}-\boldsymbol{q}_{b}\right)+p \boldsymbol{n}_{z} \\
\rho H\left(\boldsymbol{q}-\boldsymbol{q}_{b}\right)+p \boldsymbol{q}_{b}
\end{array}\right]
$$

$$
\boldsymbol{G}=\left[\begin{array}{c}
0 \\
\rho(\boldsymbol{\omega} \times \boldsymbol{q})_{x} \\
\rho(\boldsymbol{\omega} \times \boldsymbol{q})_{y} \\
\rho(\boldsymbol{\omega} \times \boldsymbol{q})_{z} \\
0
\end{array}\right] \quad \boldsymbol{F}_{v}=\left[\begin{array}{c}
0 \\
\tau_{x x} \boldsymbol{n}_{x}+\tau_{x y} \boldsymbol{n}_{y}+\tau_{x z} \boldsymbol{n}_{z} \\
\boldsymbol{\tau}_{x y} \boldsymbol{n}_{x}+\tau_{y y} \boldsymbol{n}_{y}+\tau_{y z} \boldsymbol{n}_{z} \\
\tau_{x z} \boldsymbol{n}_{x}+\tau_{y z} \boldsymbol{n}_{y}+\tau_{z z} \boldsymbol{n}_{z} \\
\psi_{x} \boldsymbol{n}_{x}+\psi_{y} \boldsymbol{n}_{y}+\psi_{z} \boldsymbol{n}_{z}
\end{array}\right]
$$

式中: $\rho$ 和 $E$ 为流体密度和总能; $u, v$ 和 $w$ 分别为旋 转坐标系下绝对速度的 3 个分量; $\boldsymbol{q}$ 和 $\boldsymbol{q}_{b}$ 为绝对速 度和坐标系运动速度 $; \boldsymbol{\omega}$ 和 $p$ 为角速度矢量和压力 $\boldsymbol{n}_{x}, \boldsymbol{n}_{y}$ 和 $\boldsymbol{n}_{z}$ 为绝对坐标系下坐标轴方向的单位向 量; $\tau_{i j}$ 为黏性应力张量; $k$ 和 $T$ 为热传导系数和 温度。

本文使用 SST 湍流模型, 基于 MRF 方法求解雷 诺平均 Navier-Stokes 方程来模拟流场。图 5 给出了 螺旋桨气动评估的计算域、边界条件设置及近壁面 网格示意图,整个计算区域由旋转域和静止域组成。 为了降低计算成本,计算区域设为直径为 $23.3 D$ 的 圆柱体的六分之一, 在计算域的两侧设置旋转周期 边界。

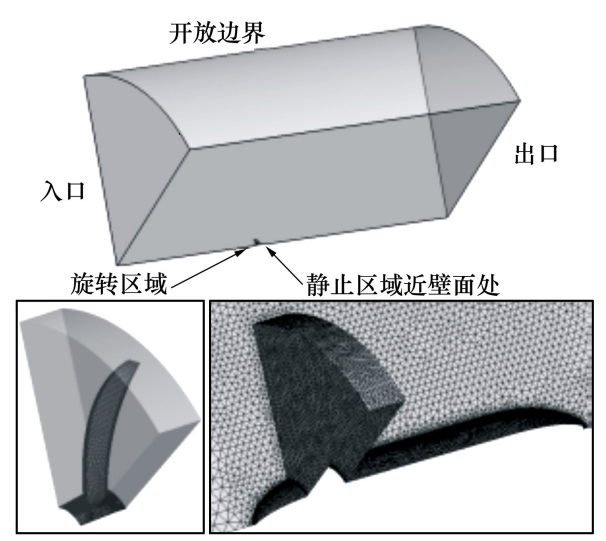

图 5 计算区域、边界条件及近壁面网格

\section{2 噪声评估}

本文采用 Hanson 等 ${ }^{[22]}$ 提出的厚度和载荷噪声 预测方法进行噪声求解。在 2017 年, Kotwicz 等 ${ }^{[16]}$ 对比早期的螺旋桨噪声预测模型, 其中包括采用 FW-H 方程的噪声预测方法, 发现在预测纯音噪声 峰值时,计算成本相对较小的 Hanson 模型计算得到 
的多个算例与实验值的平均误差最小,约有 $7.2 \mathrm{~dB}$, 适合应用于设计及优化研究工作中。

本文根据文献 [23] 对 NASA 的 SR-2 螺旋桨进 行几何造型, 表 1 给出了定义 SR-2 螺旋桨的弦长、 扭转角和翼型, 其中扭转角为各个剖面桨叶角与基 准桨叶角的差值, 取 $75 \%$ 位置处翼型桨叶角为基 准, 其数值为 $21^{\circ}$ 。成型后的模型如图 6 所示, 其桨 直径为 $0.591 \mathrm{~m}$ 。数值模拟采用海平面高度下的大 气参数, 设置来流马赫数为 0.18 , 螺旋桨转速为

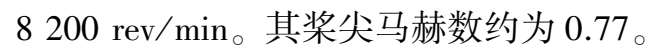

\section{表 1 SR-2 螺旋桨成型参数}

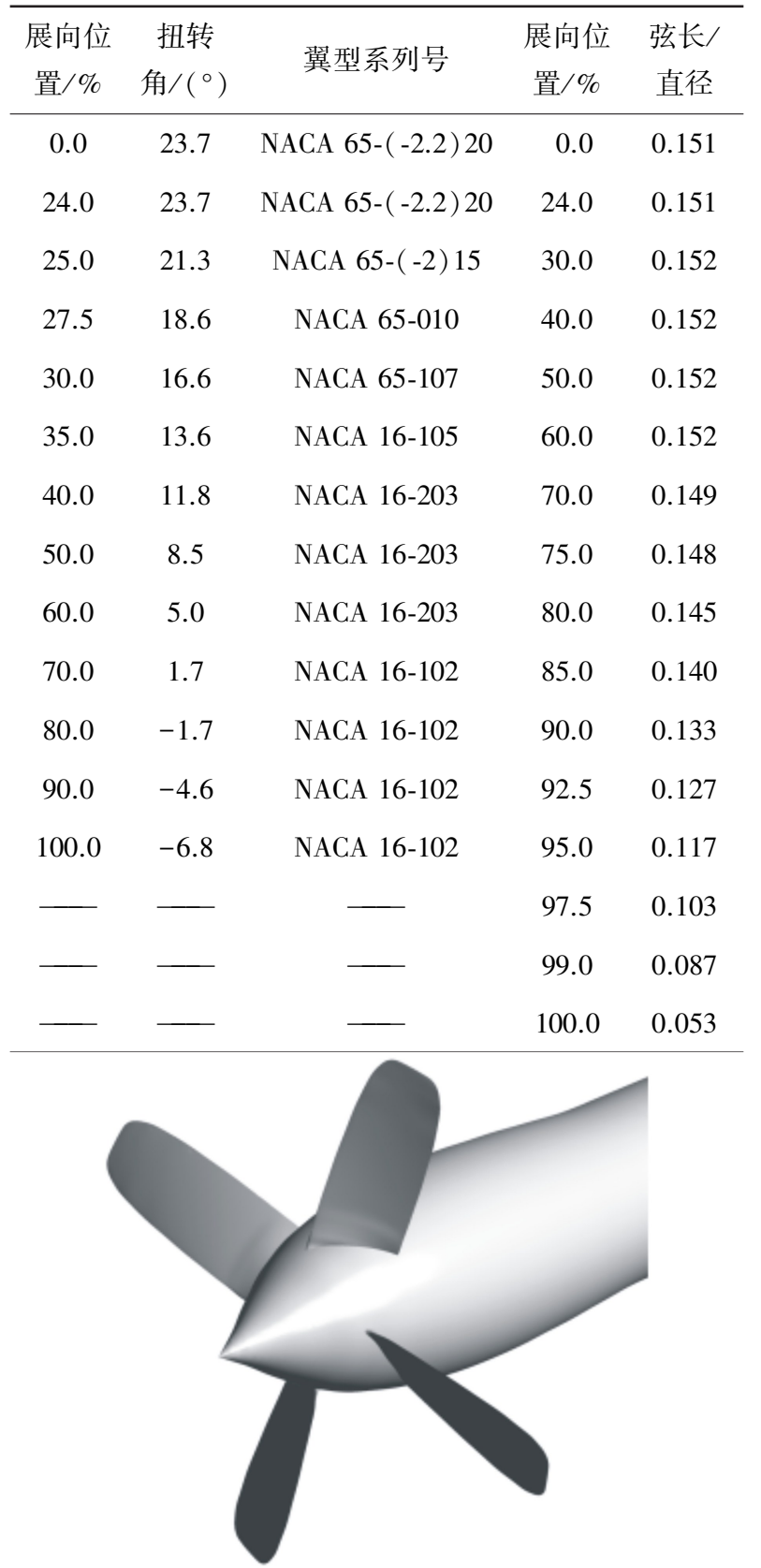

采用 RANS 方法进行气动计算。将 RANS 方程 计算得到的叶片表面网格格心处的定常压力、速度、 网格单元格心坐标、单元面积及网格单元法矢作为 Hanson 噪声模型的输人, 评估了文献中各个监测点 的噪声值。图 7 为不同噪声求解器及实验得到的噪 声指向性, 图中星形为 Hanson 噪声模型计算结果, 其气动数据通过叶素动量理论获得; 三角形为 Smart Rotor 计算结果, 采用势流和自由浴方法进行气动计 算,采用 FW-H 方程进行噪声求解; 方形为 STAR$\mathrm{CCM}+$ 商业软件计算结果, 采用 URANS 计算流场, 采用 FW-H 方程进行噪声求解 ${ }^{[16]}$; 圆形为实验结 果,菱形为本文结合 RANS 和 Hanson 模型计算得到 的结果。本文计算得到的指向性与文献 Hanson 模 型存在一定的偏差, 这是因为本文采用的 Hanson 模 型输人为精度更高的定常 RANS 方程计算结果, 而 文献中采用的是有诸多假设的叶素动量理论。本文 计算结果与其他求解器相当甚至更优, 且与实验相 符较好, 这说明采用的噪声评估手段具有一定的可 信度, 可以应用于优化设计中。

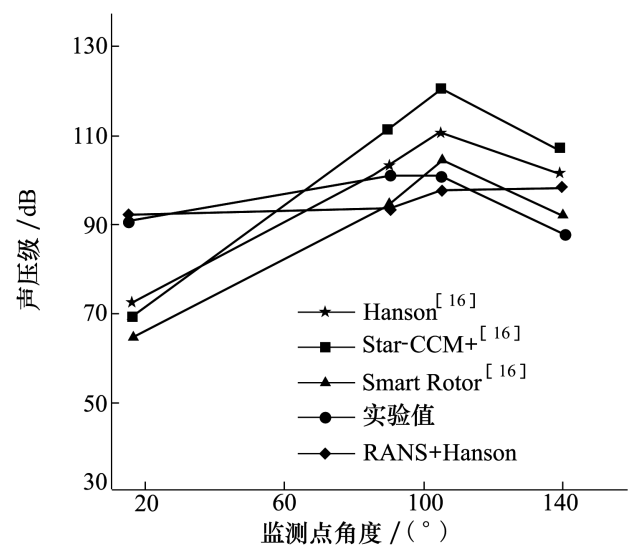

a）叶片通过频率噪声指向性

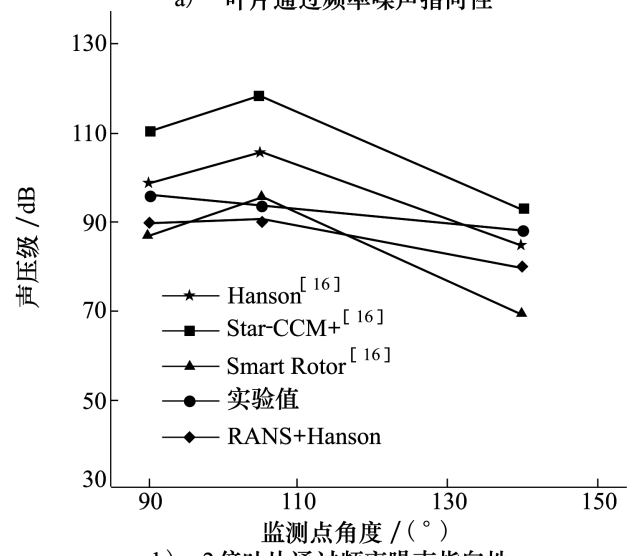

b） 2 倍叶片通过频率噪声指向性 
为考虑螺旋桨前后多个监测点叶片通过频率 (blade passing frequency, BPF) 的噪声值, 在直径为 $5 D$ 的半圆上等距选取 50 个监测点, 如图 8 所示。 50 个监测点 BPF 的声压级通过 (9) 式叠加得到优 化中判断螺旋桨噪声性能的指标

$$
L_{\text {opt }}=10 \lg \sum_{i=1}^{50} 10^{L_{i} / 10}
$$

式中, $L_{i}$ 为第 $i$ 个监测点处的声压级。

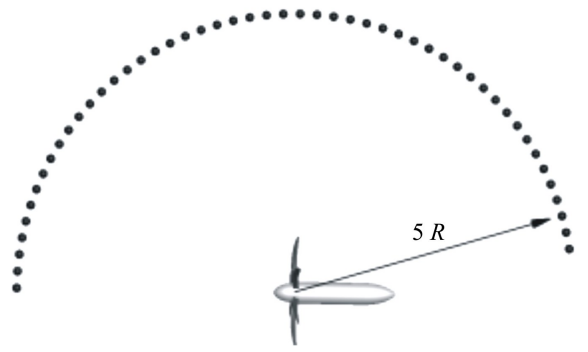

图 8 监测点位置示意图

\section{3 基于 Kriging 代理模型的 NSGAII 算法}

本文采用 NSGAII 算法进行优化搜索, 该算法 由 Deb 在 Srinivas 和 Deb 提出的 NSGA 算法 ${ }^{[25]}$ 基础 上发展而来 ${ }^{[25]}$ 。

考虑到优化过程中大量的气动性能和噪声评估 成本, 采用拉丁超立方方法随机生成涉及几何变形 的 12 个设计变量的初始种群, 并对初始种群的个体 进行气动和噪声评估, 构建设计变量、推力系数、功 率系数和 (9) 式所示的声压级样本, 继而利用该样

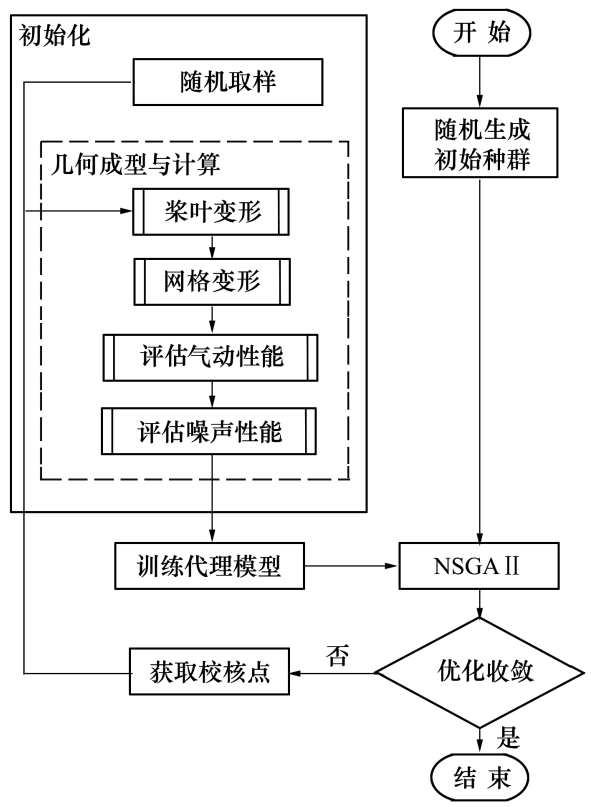

图 9 优化流程图
本训练 Kriging 代理模型。为了提高代理模型预测 的精度, 每代挑选出 Pareto 前缘上一定数目的个体 进行数值计算并加到训练样本中重新训练代理模 型。当校验点数小于 Pareto 前缘个体数时, 优先选 取拥挤度较大的个体。反之, 则在下一个非支配等 级上选取。图 9 为本文优化设计的框架。

\section{3 优化结果}

考虑气动与噪声性能的螺旋桨多目标优化设计 问题可描述为

$$
\left\{\begin{array}{l}
\text { 目标: } \min \left(C_{P}, L_{\mathrm{opt}}\right) \\
\text { 约束: }\left\{\begin{array}{l}
C_{T} \geqslant C_{T, \text { initial }} \\
t_{i}=t_{i, \text { initial }} \\
\boldsymbol{x}_{l} \leqslant \boldsymbol{x} \leqslant \boldsymbol{x}_{u} \\
x_{i, l}=-5^{\circ}, x_{i, u}=5^{\circ} \\
x_{i+6, l}=0.5, x_{i+6, u}=1.5 \\
i=1,2, \cdots, 6
\end{array}\right.
\end{array}\right.
$$

式中: $C_{T, \text { initial }}$ 和 $t_{i, \text { initial }}$ 分别为初始构型单个叶片的 推力系数和各个展向截面处翼型的最大厚度; $\boldsymbol{x}$ 为 设计变量, 其上下限分别记作 $\boldsymbol{x}_{l}$ 和 $\boldsymbol{x}_{u}$ 。 (11) 式给出 了单个叶片推力系数、功率系数和效率的计算公式, 其中 $n, \lambda, V$ 和 $\rho$ 分别为单位时间内转数、前进比、来 流速度和密度。表 2 给出了数值模拟的参数。

$$
\begin{gathered}
C_{T}=\frac{T}{\rho_{\infty} \cdot n^{2} \cdot D^{4}} \\
C_{P}=\frac{P}{\rho_{\infty} \cdot n^{3} \cdot D^{5}} \\
\eta=\lambda \frac{C_{T}}{C_{P}} \\
\lambda=\frac{V}{n \cdot D}
\end{gathered}
$$

表 2 数值模拟参数

\begin{tabular}{cc}
\hline 参数名 & 数值 \\
\hline 高度 $/ \mathrm{m}$ & 0 \\
前进比 & 1.734 \\
来流马赫数 & 0.2 \\
来流密度 $/\left(\mathrm{kg} \cdot \mathrm{m}^{-3}\right)$ & 1.225 \\
来流静温 $/ \mathrm{K}$ & 288.15 \\
转速 $/\left(\mathrm{r} \cdot \mathrm{s}^{-1}\right)$ & 53.28 \\
来流静压 $/ \mathrm{Pa}$ & 101325 \\
\hline
\end{tabular}


优化收玫后, 将样本点和每代重新计算的个体 组合成新的种群,对该种群进行非支配关系排序, 确 定种群的 Pareto 前缘, 如图 10 所示。图中菱形的可 行解为满足约束的个体, 小号方形的不可行解为违 反推力约束但相对于初始构型的绝对误差在 0.001 以内的个体, 大号方形点为初始构型, 实线为 Pareto 前缘。从图 10a) 中, 可以看到 Pareto 前缘位于初始
点的下方, 相比于噪声上的收益, 气动性能提升较 小, 这也说明初始构型具有较好的气动特性。

从 Pareto 前缘上挑选出气动性能最优、噪声性 能最优和兼顾两者的个体, 分别记为 $\mathrm{A} 、 \mathrm{~B}$ 和 $\mathrm{C}$, 如 图 10b) 所示, 同时记初始构型为 $\mathrm{D}$ 。表 3 给出了 4 个构型的计算结果。

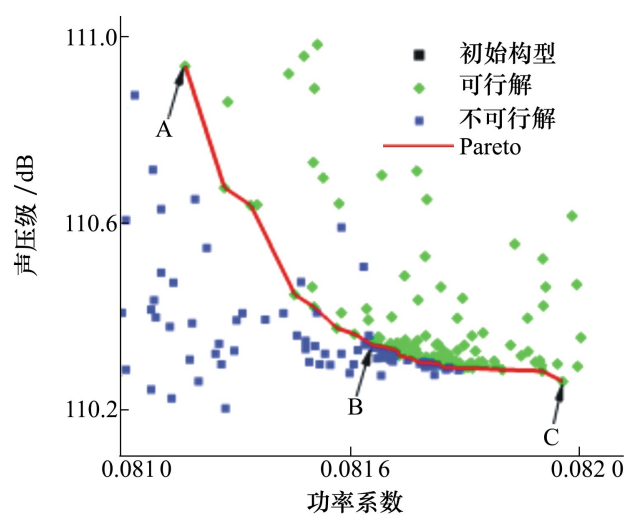

b) Pareto 前缘放大图

a) 可行解、不可行解、初始值及 Pareto 前缘整体分布图

图 10 可行解、不可行解、初始值及 Pareto 前缘分布图

表 3 优化构型及初始构型计算结果

\begin{tabular}{ccccc}
\hline 标号 & $C_{P}$ & $C_{T}$ & $L_{\text {op }} / \mathrm{dB}$ & $\eta / \%$ \\
\hline $\mathrm{A}$ & 0.08131 & 0.03861 & 110.94 & 82.345 \\
$\mathrm{~B}$ & 0.08163 & 0.03861 & 110.34 & 82.012 \\
$\mathrm{C}$ & 0.08197 & 0.03862 & 110.26 & 81.705 \\
$\mathrm{D}$ & 0.08134 & 0.03861 & 111.12 & 82.310 \\
\hline
\end{tabular}

从表 3 中可以看到, 构型 $\mathrm{A}$ 对比构型 $\mathrm{D}$, 噪声降 低了 $0.18 \mathrm{~dB}$, 效率提高了 $0.03 \%$, 气动与噪声性能
提升较低, 这是因为初始构型气动性能较优, 难以优 化得到在气动及噪声性能上都有明显提升的构型。 构型 $\mathrm{B}$ 和 $\mathrm{C}$ 气动性能略有降低, 但气动效率降低不 超过 $0.7 \%$, 此时噪声指标分别降低了 $0.78 \mathrm{~dB}$ 和 $0.86 \mathrm{~dB}$ 。相对于气动性能, 噪声性能是本文更关心 的指标, 所以允许较小的气动性能损失来换取更好 的噪声特性。图 11 给出了构型 A, B, C 和 D 厚度噪 声、载荷噪声和总噪声的指向性图, 其中横坐标为监 测点偏离来流方向的角度。

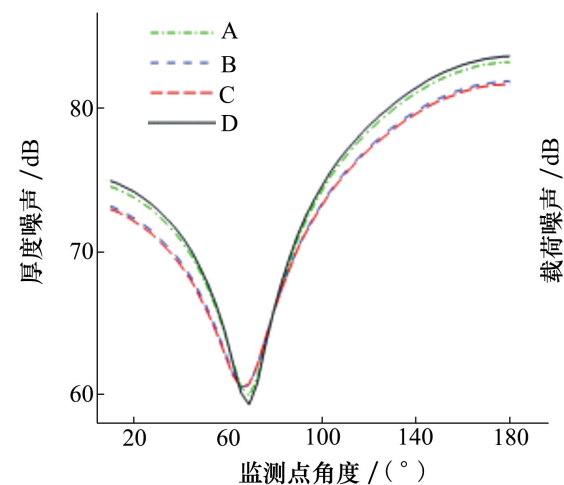

a) 厚度噪声

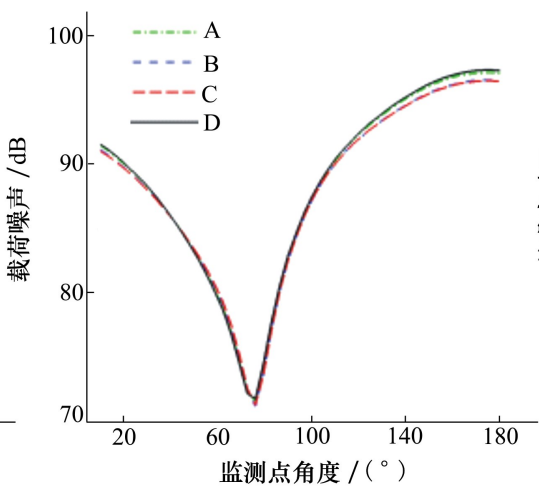

b) 载荷噪声

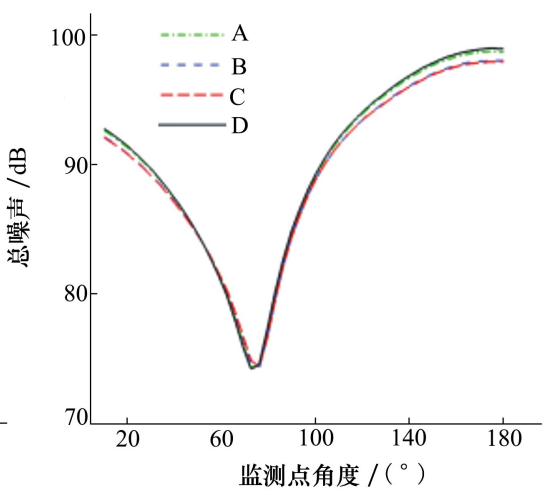

c) 总噪声

图 11 构型 A, B,C 和 D 的噪声指向性 
从图 11 中可以看到, 对比初始构型 $\mathrm{D}$, 构型 $\mathrm{A}$, $\mathrm{B}$ 和 $\mathrm{C}$ 的噪声值在螺旋桨前后大多数监测点处都有 下降, 降噪量依次递增。对比图 11a) 11c) 的噪声 值, 可以发现载荷噪声是主要的噪声源, 构型 A, B 和 $\mathrm{C}$ 虽然多数监测点处载荷噪声有所降低, 但降低 量较小, 厚度噪声降低明显。图 12 给出了各个构型 对比构型 $\mathrm{D}$ 在监测点处的降噪量。从图 12 中可以 看到, 优化构型 $\mathrm{B}$ 和 $\mathrm{C}$ 大多数监测点的厚度噪声降
噪量在 $1.5 \mathrm{~dB}$ 以上, 最大降噪量近 $2 \mathrm{~dB}$, 如图 12a) 所示。多数监测点的载荷噪声降噪量约为 $0.5 \mathrm{~dB}$ 左右, 最大降噪量约 $0.8 \mathrm{~dB}$, 如图 12b) 所示。从图 $12 \mathrm{c}$ ) 中可以看到, 构型 $\mathrm{B}$ 和 $\mathrm{C}$ 大多数监测点的总噪 声降低在 $0.5 \mathrm{~dB}$ 以上,构型 $\mathrm{C}$ 在螺旋桨正前方监测 点的降噪量可达 $1 \mathrm{~dB}$, 构型 $\mathrm{B}$ 可达 $0.8 \mathrm{~dB}$, 构型 $\mathrm{A}$ 约为 $0.25 \mathrm{~dB}$ 。

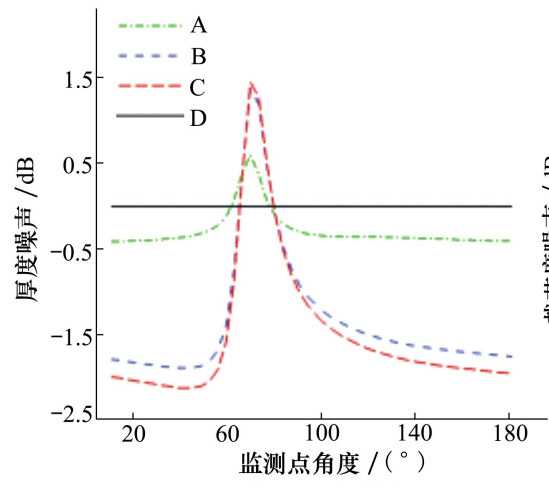

a) 厚度噪声

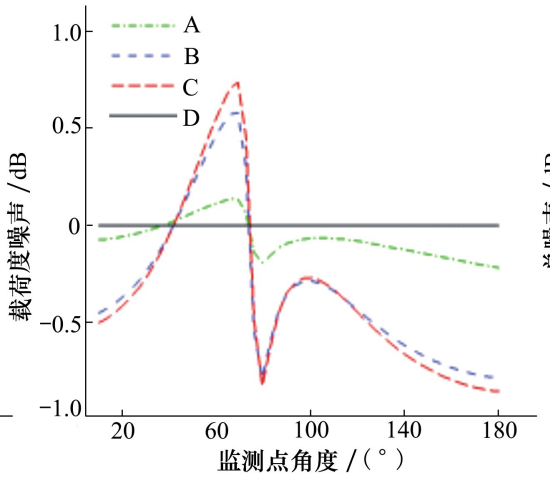

b) 载荷噪声

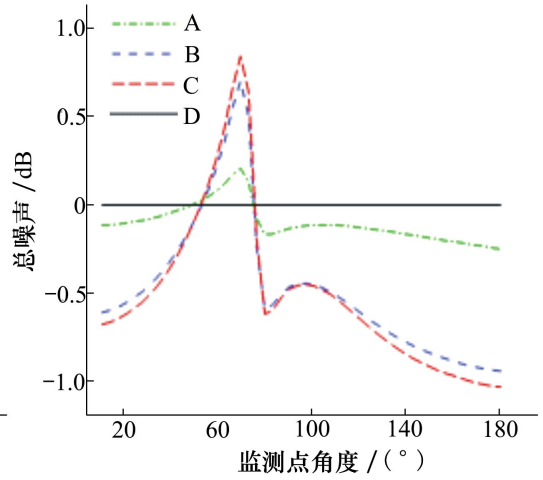

c) 总噪声

图 12 构型 $\mathrm{A}, \mathrm{B}, \mathrm{C}$ 和 $\mathrm{D}$ 各个监测点相对于构型 $\mathrm{D}$ 的降噪量

为了进一步分析噪声下降的原因, 图 13 给出了 构型 A, B , C 和 D 各个剖面处的翼型, 除叶根和叶尖 外的 7 个翼型分别通过圆柱面与桨叶相交得到。

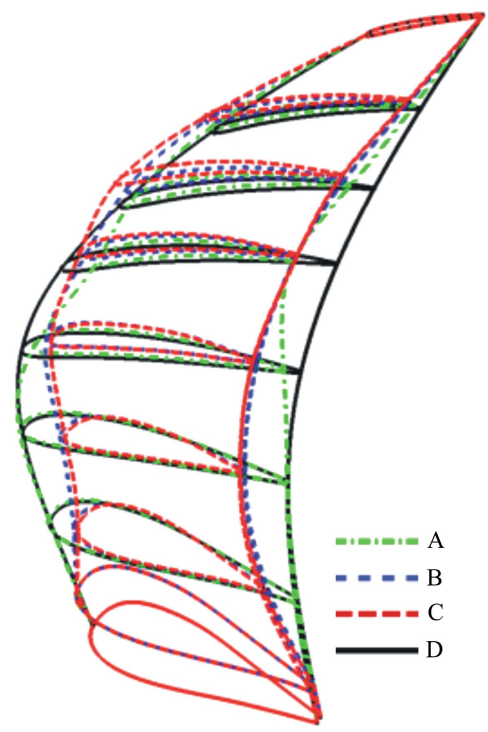

图 13 构型 A,B,C 和 D 翼型对比

从图 13 中可以看到, 构型 $\mathrm{B}$ 和 $\mathrm{C}$ 剖面翼型弦 长减小, 扭转角增大。Hanson ${ }^{[26]}$ 曾提出在不考虑厚 度分布的情形下, 环向截面处翼型辐射的厚度噪声
近似与 $t_{b} B_{D}^{2}$ 成正比, 其中 $t_{b}$ 为翼型最大厚度与弦长 的比值, $B_{D}$ 为弦长与螺旋桨直径的比值。因为优化 保证翼型的最大厚度不变, 所以厚度噪声近似与弦 长成正比。因此,优化构型 $\mathrm{B}$ 和 $\mathrm{C}$ 的厚度噪声有显 著的降低,如图 12a) 所示。同时为保持足够的推 力, 各个剖面的扭转角增大补偿弦长减小导致的推 力损失。构型 $\mathrm{A}$ 几何变化类似, 但是翼型弦长和扭 转角变化相对较小。

\section{4 结 论}

本文基于 NFFD 方法、Hanson 噪声模型等方 法, 建立了考虑气动性能与噪声性能的螺旋桨优化 设计框架。采用该优化框架对某民航客机螺旋桨进 行了优化设计,得到以下结论:

1) 基于 NFFD 方法和 RBF 方法可以有效地实 现螺旋桨外形参数化和网格生成。

2) Hanson 噪声模型可以以较低的计算成本, 很好地预测螺旋桨的纯音噪声, 适合应用于螺旋桨 的工程设计及优化问题。

3) 基于 Kriging 代理模型与 NSGAII 算法建立 的从 Pareto 前缘挑选个体重新训练代理模型的优化 
框架具有较高的优化效率, 大大降低了优化成本。

4) 综合噪声性能较好的优化构型特征为:螺旋 桨桨叶各个剖面弦长减小, 降低厚度噪声; 扭转角增 大, 以保证足够的推力, 同时改变叶片载荷分布, 降 低载荷噪声。厚度噪声的降噪量更为显著。然而,
优化选取的螺旋桨和计算状态下, 载荷噪声为主要 噪声源, 厚度噪声相对较小。后续可以考虑针对载 荷噪声, 减小弦长扰动范围, 对各个剖面翼型及扭转 角进行优化设计。

\section{参考文献:}

[ 1] PAGANO A, FREDERICo L, BARBARINo M, et al. Multi-Objective Aeroacoustic Optimization of an Aircraft Propeller[C] // 12th AIAA/ISSMO Multidisciplinary Analysis and Optimization Conference, 2008

[2] MARINUS B G, ROGER M, VAN DEN BRAEMbUSSCHE R A, et al. Multidisciplinary Optimization of Propeller Blades: Focus on the Aeroacoustic Results[C] //17th AIAA/CEAS Aeroacoustics Conference, 2011

[3] Canard-Caruana S, Le tallec C, Beaumier P, et al. Anibal: a New Aero-Acoustic Optimized Propeller for Light Aircraft Applications[C] //19th AIAA Aviation Technology, Integration and Operations Conference and Aircraft Noise and Emisions Reduction Symposium, 2010

[4] 王博,招启军,徐国华. 悬停状态直升机桨叶扭转分布的优化数值计算 [J]. 航空学报, 2012,33(7) : 1163-1172 WANG Bo, ZHAO Qijun, XU Guohua. Numerical Optimization of Helicopter Rotor Twist Distribution in Hover[J]. Acta Aeronautica et Astronautica Sinica, 2012,33(7): 1163-1172 (in Chinese)

[5] 招启军,蒋霜,李鹏, 等. 基于 CFD 方法的倾转旋翼/螺旋桨气动优化分析 [ J]. 空气动力学报, 2017,35(4) : 544-553 ZHAO Qijun, JIANG Shuang, LI Peng, et al. Aerodynamic Optimization Analyses of Tiltrotor/Propeller Based on CFD Method [J]. Acta Aerodynamic Sinica, 2017,35(4) : 544-553 (in Chinese)

[6] 郭旺柳, 宋文萍, 许建华, 等. 旋翼桨尖气动/降噪综合优化设计研究 $[\mathrm{J}]$. 西北工业大学学报, 2012,30(1) : 73-79 GUO Wangliu, SONG Wenpeng, XU Jianhua, et al. An Effective Aerodynamic/Acoustic Optimization of Blade Tip Planform for Helicopter Rotors $[\mathrm{J}]$. Journal of Northwestern Polytechnical University, 2012,30(1) : 73-79 (in Chinese)

[7] 朱正,招启军. 低 HSI 噪声旋翼桨尖外形优化设计方法 $[J]$. 航空学报, 2015,36(5) : 1442-1452

ZHU Zheng, ZHAO Qijun. Optimization Design Method for Rotor Blade-Tip Shape with Low HIS Noise Character $[\mathrm{J}]$. Acta Aeronautica et Astronautica Sinica, 2015, 36(5): 1442-1452 (in Chinese)

[8] GUTIN L. On the Sound Field of a Rotating Propeller[R]. NACATM-1195, 1948

[9] DEMING A F. Noise from Propellers with Symmetrical Sections at Zero Blade Angle[R]. NACA TN-679, 1937

[10] GARRICK L E, WATKINS. A Theoretical Study of the Effect of Forward Speed on the Free-Space Sound-Pressure Field around Propellers[R]. NACA Report 1198, 1953

[11] ARNOLDI R A. Propeller Noise Caused by Blade Thickness [R]. United Aircraft Corporation Research Department Report R0896-1, 1956

[12] ARNOLDI R A. Near Field Computations of Propeller Blade Thickness Noise[R]. United Aircraft Corporation Research Department Report R-0896-2, 1956

[13] BARRY F W, MAGLIOZZI B. Noise Detectability Prediction Method for Low Tip Speed Propellers[R]. Hamilton Standard Division TR-71-37, 1971

[14] HANSON D B. Helicoidal Surface Theory for Harmonic Noise of Propellers in the Far Field[J]. AIAA Journal, 1980, 18( 10): 1213-1220

[15] HANSON D B. Sound from a Propeller at Angle of Attack: a New Theoretical Viewpoint $[\mathrm{J}]$. Proceedings of the Royal Society of London. Series A: Mathematical and Physical Sciences, 1995, 449(1936) : 315-328

[16] KOTWICZ H M T, FESZTY D, MESLIOUI S A, et al. Applicability of Early Acoustic Theory for Modern Propeller Design[C] //23rd AIAA/CEAS Aeroacoustics Conference, 2017

[17] KOTWICZ H M T, FESZTY D, MESLIOUI S A, et al. Evaluation of Acoustic Frequency Methods for the Prediction of Propeller Noise[ J]. AIAA Journal, 2019, 57(6): 2465-2478

[18] SEDERBERG T W, PARRY S R. Free-Form Deformation of Solid Geometric Models[C] // Proceedings of the 13th Annual Con- 
ference on Computer Graphics and Interactive Techniques, 1986: 151-160

[19] LAMOUSIN H J, WAGGENSPACK JR W N. NURBS-Based Free-Form Deformations[J]. IEEE Computer Graphics and Applications, $1994(6): 59-65$

[20] BOER A D, VON DER SCHOOT M S, BIJL H, Mesh Deformation Based on Radial Basis Function Interpolation[J]. Computers \& Structures, 2007, 85(11): 784-795

[21] 徐家宽,白俊强,黄江涛,等. 考虑螺旋桨滑流影响的机翼气动优化设计研究 $[\mathrm{J}]$. 航空学报, 2014,35(11):2910-2920

XU Jiakuan, BAI Junqiang, HUANG Jiangtao, et al. Study of Aerodynamic Optimization Design of Wing under the Interaction of Propeller Slipstream [J]. Acta Aeronauticaet Astronautica Sinica, 2014,35(11) : 2910-2920 (in Chinese)

[22] HANSON D B, PARZYCH D J. Theory for Noise of Propellers in Angular Inflow with Parametric Studies and Experimental Verification[R]. NASA CR-1993-4499, 1993

[23] HAMBREY J. Computational Aeroacoustic Prediction of Propeller Noise Using Grid-Based and Grid-Free CFD Methods[D]. Ottawa, Carleton University, 2016

[24] SRINIVAS N, DEB K. Multi-Objective Function Optimization Using Non-Dominated Sorting Genetic Algorithms[J]. IEEE Trans on Evolutionary Computation, 1995, 2(3) : 221-248

[25] DEB K, PRATAP A, AGARWAL S, et al. A Fast and Elitist Multiobjective Genetic Algorithm: NSGA- II [J]. IEEE Trans on Evolutionary Computation, 2002, 6(2): 182-197

[26] HANSON D B. Influence of Propeller Design Parameters on Far-Field Harmonic Noise in Forward Flight [ J]. AIAA Journal, $1980,18(11): 1313-1319$

\title{
Aerodynamic and Aeroacoustic Optimization of Propeller Based on Hanson Noise Model
}

\author{
SONG Xiang, YU Peixun, BAI Junqiang, HAN Xiao, PENG Jiahui \\ ( School of Aeronautics, Northwestern Polytechnical University, Xi'an 710072, China)
}

\begin{abstract}
Aiming at the multi-objective optimization design problem of propeller aerodynamics and noise, the three-dimensional geometric deformation of the whole blade is carried out by the free-form surface deformation method based on non-uniform rational B-spline. In order to save the calculation cost of optimization, the RANS method and the Hanson model are combined to predict pure tone noise, and the prediction accuracy is comparable to the accuracy of the FW-H equation coupled with URANS method. Kriging surrogate model and non-dominated sorting genetic algorithm are used to search for optimal value, and a multi-objective optimization design framework for propeller aerodynamics and noise is established. This method is used to optimize the blade shape of a passenger airliner propeller, and the airfoil torsion angle and chord length of different positions are optimized as design variables. Compared with the basic blade, the noise value of the axial monitoring point near the cruise configuration under the wind tunnel experiment condition is reduced by about $0.25 \mathrm{~dB}$ at the same time as the power is reduced. In the case of a slight increase in power, the noise is reduced by about $1 \mathrm{~dB}$.
\end{abstract}

Keywords : free-form surface deformation method (NURBS-FFD); multiple reference frame (MRF) ; Hanson noise model; non-dominated sorting algorithm (NSGAII); Kriging surrogate model 